Chapman University

Chapman University Digital Commons

Physical Therapy Faculty Articles and Research

Physical Therapy

4-9-2018

\title{
Effects of Load Carriage and Surface Inclination on Linear and Non-Linear Postural Variability
}

\author{
Rahul Soangra \\ ChapmanUniversity, soangra@chapman.edu
}

Hema Bhatt

G.B. Pant University of Agriculture \& Technology

Ehsan Rashedi

Follow this and additional works at: https://digitalcommons.chapman.edu/pt_articles

Part of the Medical Anatomy Commons, Medical Biophysics Commons, Medical Physiology Commons, Musculoskeletal, Neural, and Ocular Physiology Commons, $\underline{\text { Musculoskeletal System }}$ Commons, and the Physiological Processes Commons

\section{Recommended Citation}

Soangra R, Bhatt H, Rashedi E. Effects of load carriage and surface inclination on linear and non-linear postural variability. Saf Sci. 2018;110(A):427-437. doi: 10.1016/j.ssci.2018.03.019

This Article is brought to you for free and open access by the Physical Therapy at Chapman University Digital Commons. It has been accepted for inclusion in Physical Therapy Faculty Articles and Research by an authorized administrator of Chapman University Digital Commons. For more

information, please contact laughtin@chapman.edu. 


\title{
Effects of Load Carriage and Surface Inclination on Linear and Non- Linear Postural Variability
}

\author{
Comments \\ NOTICE: this is the author's version of a work that was accepted for publication in Safety Science. Changes \\ resulting from the publishing process, such as peer review, editing, corrections, structural formatting, and \\ other quality control mechanisms may not be reflected in this document. Changes may have been made to this \\ work since it was submitted for publication. A definitive version was subsequently published in Safety Science, \\ volume 110, part A, in 2018. DOI: 10.1016/j.ssci.2018.03.019 \\ The Creative Commons license below applies only to this version of the article.
}

\section{Creative Commons License}

\section{(c) 1 (1) 90}

This work is licensed under a Creative Commons Attribution-Noncommercial-No Derivative Works 4.0 License.

\section{Copyright}

Elsevier 


\title{
Effects of Load Carriage and Surface inclination on linear and non-linear Postural Variability
}

\begin{abstract}
Understanding of inclined-work-related risk of falls and developing novel practical engineering controls for reducing this risk of falls among hilly working population remains in high demand. Standing on sloped surfaces provides a unique environment for examining the biomechanics and neural control of standing. The present investigation examined the variability of postural signals when standing on inclined surfaces and with load carriages by linear and nonlinear analysis. The purpose of this study was to determine if the sloped surface deteriorated in postural stability among healthy individuals with two distinctive kinds of load carriage methods head versus posterior load carriage. We also examined the effects of distinct magnitudes of load on these conditions. Postural control was assessed objectively using forceplates and subjectively through perceived stability ratings. The results indicate significant differences in mediolateral COP ranges, COP velocities and COP area with interaction in surface inclinations and methods of load carriage. We found that head load carriage when standing on uphill afflicted and engendered increased balance deterioration in healthy young subjects. We also found the significantly lower complexity of postural signals for head load carriage as measured by entropy. Apropos to this mean subjective perceived rating was also least in this load-bearing condition. Understanding these underlying mechanisms of postural control with load carriage strategies in humans could productively help in developing efficacious preventive strategies to reduce the incidence of falls from inclined slopes.
\end{abstract}

\section{Introduction}

Load carriage is one of the most physically demanding occupational tasks contributing to injury and falls as well as a major risk factor for loss of balance (Thurmon E Lockhart 2013, Yeoh, Lockhart et al. 2013, Muslim and Nussbaum 2015). Load carriage is involved in many industries like construction, agriculture, transportation, and warehousing; where the worker often carries loads not only at flat surfaces but also at various degrees of surface inclinations. Negotiated standing on inclined surfaces (non-neutral posture) during load carriage is a common challenge faced by many working populations and thereby places a specific demand on the neuromuscular control system. Operating in such an environment (i.e. construction and/or roof work, etc.) is challenging to the postural control system, individuals are at an increased risk of loss of balance, and potentially a subsequent fall (Sun, Walters et al. 1996, S. Redfern and DiPasquale 1997, Gauchard, Lascombes et al. 2001, Redfern, Cham et al. 2001, Simeonov, Hsiao et al. 2003, Wade, Weimar et al. 2004, Frames, Soangra et al. 2013). Typical injuries related to falling from inclined planes with height (example roof or hills) are found to be extremely severe and require long periods of medical treatment and costs(Gillen, Faucett et al. 1997). Laborers and freight, stock, and material movers, who often carry the load at various surface inclinations, have been reported as the occupations with the highest number of days-away-from-work cases reported in 2013(BLS 2014-2015).

In many developing countries, people routinely carry extraordinary loads supported by their heads (Head Load carriage: HLC) or back (Posterior Load Carriage: PLC)(Chow, Li et al. 2011). Further, PLC and HLC among these workers are typically without the use of a backpack or other assistive devices (Figure1). PLC and HLC performance involves the nonneutral trunk poses which when adopted may increase postural sway i.e. affecting the postural stability of the person. Such traditional load carriage methods are prevalent in both domestic and occupational settings. For instance, workers at the grain storage depots, construction sites and many other places of trade and commerce, use these methods to carry the load that may be as high as $100 \mathrm{~kg}\left(\mathrm{Nag}^{*}\right.$ and Sen 1979). Both men and women laborers are engaged in such hefty load carriage activities. For example, in the 
underdeveloped areas of the hills in the Himalayan regions, women convey a ponderous load daily for their livelihood, transporting food, fuel, and fodder on risky uphill and downhill slopes (figure 1). Carrying a load on inclined uphill and downhill slopes often results in loss of balance and fall accidents in many outdoor work environments. Carrying an external load on the back shifts the system's center of mass posteriorly, and individuals typically adopt a forward lean to maintain balance (Goodgold, Mohr et al. 2001, Grimmer, Dansie et al. 2002, Hong and Cheung 2003). Unwittingly standing on inclined planes predisposes to fall by itself (Cham and Redfern 2002) regarding its adaptation when the load is carried at such inclined surfaces. Biomechanics research has focused largely on walking over horizontal (Perry, k et al. 1992), with less attention paid to inclined surfaces(Leroux, Fung et al. 2002). For instance, there are few studies that have focused on downhill walking (Sun, Walters et al. 1996, S. Redfern and DiPasquale 1997), uphill walking (Kang, Chaloupka et al. 2002, Noble and Prentice 2008) and cross-slope walking (Damavandi, Dixon et al. 2012). In seminal work on the load carriage, researchers have concentrated mostly on muscle activity patterns (Thomas, Stein et al. 1987) and physiological strain variables (Bhambhani and Maikala 2000). The flurry of research surrounding traditional load carriage methods has been limited to experimental studies assessing the physiological cost (Minetti, Formenti et al. 2006, Lloyd, Parr et al. 2010), gait (Heglund, Willems et al. 1995), a load coupled to the trunk in the form of vest or backpack (Birrell and Haslam 2009, Thurmon E Lockhart 2013) and ratings of perceived discomfort (Lloyd, Parr et al. 2010). Seminal work by researchers (Kinoshita 1985, Goh, Thambyah et al. 1998, Chansirinukor, Wilson et al. 2001, Hong and Cheung 2003) has reported that significant postural adaptations occurred in response to load carriage.

Balance is the amount of postural sway (also called body sway) of the human body. Postural sway is the slight postural movement made by an individual in order to maintain a balanced position and is the total displacement at the center of mass relative to the base of support over time. The postural sway is by contraction and relaxation of muscle groups and is an indirect measure of the propensity for falls. Postural sway has been assessed for static balance and dynamic balance conditions, depending on whether the base is stationary or moving (such as standing or walking)(Spirduso and Asplund 1995). Predominantly, increased postural sway inferred as impaired postural control, and is associated with an increased falling risk (Fernie, Gryfe et al. 1982, Lichtenstein, Shields et al. 1988, Maki, Holliday et al. 1990, Baloh, Spain et al. 1995, Prieto, Myklebust et al. 1996). Since standing on inclines and load carriage induce deviations from natural posture, it can lead to increased stress in neck and low back(Chaffin and Anderson 1984). And prolonged working in these environments may lead to postural discomfort, increased lumbosacral forces (Goh, Thambyah et al. 1998) and muscular pain in neck, shoulder, or low back injuries(Chaffin and Anderson 1984). A greater amount of postural instability correlated with an increased risk of falling. The mass of an external load on the body (Ledin and Odkvist 1993, Holbein and Redfern 1997) and surface inclinations are the potential variables affecting body's stability limits and equilibrium.

To our best knowledge, this is the first study that specifically investigated postural stability with different methods of traditional load carriage without the use of packs or other assistive devices on inclined surfaces. Information regarding the effects of load carriage methods on balance and postural stability is sparse. Therefore the purpose of this study is to facilitate a more comprehensive understanding of the contribution of different load carriage methods at inclined surfaces to postural stability; which may help to identify effective practical interventions to facilitate injury prevention from loss of balance and associated falls while carrying the load on inclined surfaces. We hypothesized that different load carriage methods, and surface inclinations would disparately affect postural control and balance outcomes. The specific questions addressed in this study are: if there exist any mismatches between subjective perceptions of an impending loss of balance and actual (as 
measured by objective measures using forceplate- a gold standard assessment) risk of fall or steadiness? The main objective of this study was to investigate the effects of surface slope, load carriage, load magnitude and their interactions in the control and perception of standing balance in young healthy subjects. Understanding these underlying causes and effects on balance could be useful in developing efficacious preventive strategies to reduce the incidence of falls from inclined slopes.

\section{Methods}

The study recruited twenty participants (gender balanced) from Virginia Tech and the local community. Participants had no self-reported injuries, musculoskeletal disorders, neurological disorders, vestibular disease, or occurrences of falls in the past 12 months. Mean (SD) age, stature, and body mass of the male participants were 21.4 (1.5) years, $177.4(7.1) \mathrm{cm}$, and 76.1 (8.1) kg, respectively, with corresponding values of 21.4 (2.0) years, $161.8(7.7) \mathrm{cm}$, and $61.4(8.9) \mathrm{kg}$ for the female participants. Prior to data collection, participants completed informed consent procedures approved by the Virginia Tech Institutional Review Board.

Experimental design and procedures: A repeated-measures design was used, in which each participant completed load carriage tasks under several conditions, comprised of all combinations of three load magnitudes (LM), three surface inclinations (SI), and two load carriage methods (LC). Note that $3 \times 2 \times 3$ (surface inclination (uphill, downhill, level) $x$ load carriage (head, posterior) $x$ load magnitude (high, medium, no load) eighteen distinct conditions were tested. Surface Inclination angle (SI): Three inclination angles were evaluated: horizontal $\left(0^{\circ}\right)$, uphill $\left(+20^{\circ}\right)$, and downhill $\left(-20^{\circ}\right)$. This pitch angle was determined in accordance with previous investigations (Simeonov et al., 2003) and by the Occupational Safety and Health Administration (OSHA, 1995). OSHA classifies 4/12 $\left(\sim 18^{\circ}\right)$ as a low-sloped roof and 6/12 $\left(\sim 26^{\circ}\right)$ as steep-sloped. Therefore, we opted for a slope surface angle in between a low-sloped and steep-sloped angle; a pitch that workers in many industries like roof workers, commonly perform tasks on without any additional support devices. A forceplate placed at a specific inclination angle (AMTI OR6- 7-1000, Watertown, MA, USA) on a custom-made walkway at the desired angle. To capture center of pressure data, a custom-built wooden platform utilized for the experiment. A ramp with a level walking the track and $20^{\circ}$ inclined walking track was built and installed in the Occupational Ergonomics and Biomechanics Laboratory at Virginia Tech. The apparatus consisted of a level-walking track $(5.5 \mathrm{~m})$ with a force plate (Advanced Mechanical Technology (AMTI), Inc., OR6-7-1000, Watertown, MA, USA) in between. In order to simulate load carriage on slopes, a force platform mounted on an inclined wooden platform. For uphill and downhill trials, the inclined walking track consisted of combined level-inclined-level surface $(3.5 \mathrm{~m})$, with two force plates (Advanced Mechanical Technology (AMTI), Inc, OR6-7-1000, Watertown, MA, USA), one in level and one in inclined surface to measure the relevant postural parameters during the flat and inclined surface standing trials. Load Magnitude $(L M)$ : Participants carried a customized load of two weights: medium load- $7.5 \mathrm{~kg}$ and heavy load- $15 \mathrm{~kg}$. Experiment conducted in a no-load condition, was used to normalize the measures in other loading conditions. Loads designed with a bag of similar size filled with sand, paper, and pet food to make it compact. For posterior load carriage, two ropes were fixed in a way so as to simulate the way load carrying is conducted in many industries especially in developing countries (i.e., without the use of an assistive device). Load Carriage (LC): Two load carriage methods studied, i.e. HLC and PLC. During the HLC load was held on the head and both arms up supporting the load from either side. During the PLC, load held on the back, stabilized with the hands at the top of the load, so both arms up supporting the load (Figure 2).

Prior to data collection, participants completed informed consent procedures approved by the Virginia Tech Institutional Review Board. We provided standardized experimental shoes to the participants to remove effects of footwear on their stability. For acclimatization, prior trials for standing with different load conditions were conducted. 
Additionally, we collected participant's demographic data (e.g., body weight and height). Static Postural Stability: Each participant completed 15 randomly assigned trials for static postural stability, with a variation of surface, load magnitude, and mode of load carriage. During the static postural stability tasks, participants stood on a force platform (AMTI OR6-71000, Watertown, MA). Each trial lasted 75 seconds, during which participants stood on the platform with their feet together, arms by their sides (if without load) or supporting the load and head pointed straight ahead. A helper placed the load at the start and lowered the load at the end of each trial. We instructed our pparticipants to "stand as still as possible" and to think about their level of "perceived stability" during the period of the quiet standing.

Repeatability of foot placement between trials was maintained by outlining the feet on top of the force platform using tape. Between trials, we provided one minute of seated rest to reduce possible after-effects of standing with load and the development of fatigue. Figure 3 shows the experimental setup for different LC and SI.

Subjective responses of the participants: At the end of each standing and walking trial, subjects were asked to evaluate their subjective feeling of the quality of steadiness throughout that particular trial. The evaluation took the form of a score, ranging from 0 (worst) to 10 (best). Half points and quarter points allowed, to distinguish trials with small differences. To give a reference, at the beginning of the session each subject was invited to stand in two postures corresponding to extremes of the range of stability: (1) with eyes open, feet apart, grasping a solid bar in front of them; this was judged by the subjects as a very stable condition, to which 10 should be attributed, (2) with eyes closed and no support, in a one leg stance condition: this could not be maintained for extended periods of time, and was judged as a very unstable condition meriting a very low score; in the case of the subjects lowering the other leg to avoid a fall, a 0 score should be attributed to the trial. All subjects understood the instruction well.

\section{Data Collection and Dependent Variables}

Force platform data were low-pass filtered (bi-directional, 2nd order Butterworth) with cut-off frequencies of $6 \mathrm{~Hz}$, and force platform data were sampled at $120 \mathrm{~Hz}$. Relevant and stable measures of postural sway were calculated and subject to statistical analysis. These included AP and ML Sway, COP Velocity, Path Length, Circular Area and Elliptical Area. AP and ML Sway refers to COP excursions in anterior-posterior and medial-lateral directions. Each normalized parameter to values obtained in the relevant no-load condition (e.g., COP area for one participant during PLC was divided by COP area during no-load condition to obtain the normalized COP area). Furthermore, non-linear variables such as approximate entropy (ApEn), Sample Entropy (SampEn), Multiscale entropy (MSE) and Hurst selfsimilarity exponent alpha using Detrended Fluctuation Analysis (DFA) for raw mediolateral $(\mathrm{ML})$ and anterior-posterior (AP) center of pressure signals was also extracted. Approximate entropy is quantified as the negative natural logarithm of the conditional probability (CP) that a short template of data is repeated during the time series (Richman, Lake et al. 2004). Firstly, a template of length $\mathrm{m}$ data points is selected, and one identifies other templates that are arbitrarily similar to the next $m+1$ point. When points are within a tolerance $r$ of each other, where $r$ is a factor of standard deviation (SD), then these data points are considered arbitrarily similar. For each possible template, the negative logarithm of the conditional probability is calculated. If the data are ordered, then the templates of $m$ data points are often similar to $\mathrm{m}+1$ data points, CP approaches 1 , and the negative logarithm and entropy approaches 0(Richman, Lake et al. 2004). Sample Entropy, SampEn(m,r, N) depends on three parameters. The first parameter $m$ is the length of vector considered for analysis. Such that given $\mathrm{N}$ data points $\mathrm{u}(\mathrm{j}): 1 \leq \mathrm{j} \leq \mathrm{N}$, from the $\mathrm{N}-\mathrm{m}+1$ vectors $x m(i)$ for $\mathrm{i}: 1 \leq \mathrm{i} \leq \mathrm{N}-\mathrm{m}+1$ where $x m(i)=u(i+k): 0 \leq k \leq m-1$ is the vector of $m$ data points from $u(i)$ to $u(i+m-1)$. And the distance between two vectors $d[x m(i), x m(k)]$, is maximum at $|u(i+j)=u(k+j)|: 0 \leq j \leq m-1$. $\operatorname{SampEn}(m, r, N)$ is negative natural logarithm of the empirical probability that $d[x m+1(i), x m+1(k)] \leq r$ and given that $d[x m(i), x m(k)] \leq r$. Where $B$ denotes the number of pairs $x m(i), x m(k)$ such that 
$d[x m(i), x m(k)] \leq r$ and $A$ be the number of pairs of vectors $x m+1(i), x m+1(k)$ such that $d[x m+1(i), x m+1(k)] \leq r$. Then SampEn $(m, r, N)=-\operatorname{In}(A / B)($ Richman, Lake et al. 2004).

Statistics: Three-way MANOVA with subjects as the randomized effect were performed on the postural parameters from different LM, LC, and SI. Linear postural parameters comprised center of pressure (COP) velocity, the elliptical area of COP, path length of COP, the range of movements in anterior-posterior and mediolateral directions. Non-linear postural parameters comprised of ApEn, SampEn, MSE, DFA of ML and AP COP signals. Provided the MANOVA was significant, a univariate threeway ANOVA was performed on each dependent variable to determine those which possessed significant variance. If the univariate three-way ANOVA showed significance for the main effects for independent variable interaction, then trend analysis was performed as multiple comparisons using Tukey post hoc test. Statistics significance level was accepted at the 0.05 level of confidence.

\section{Results}

The primary hypothesis, that the LC, LM, and SI would influence participants balance was tested using MANOVA for dependent variables. The three-way MANOVA analysis revealed a significant in overall carriage effect (Wilk's Lambda $=0.727$, $F(5,281)=21.1, p<0.001$ ), overall surface inclination effect (Wilk's Lambda $=0.255$, $F(10,562)=55.0, p<0.001$ ) and overall load magnitude effect (Wilk's Lambda $=0.818$, $F(5,281)=12.52, p<0.001$ ) (Table 1). This significant result allowed further univariate analysis to determine which of the dependent variables were significant.

We found that SI, LC, and LM affected excursion range of COP in both medial-lateral and anterior-posterior directions (Figure 4). Interaction effects were found between LM and LC (Figure 5). Subject's elliptical area was significantly different at the level surface compared to the values obtained on inclined surfaces, and was maximum on a flat surface and decreased at inclined surface, ie $25 \%$ and $32 \%$ at downhill and uphill condition respectively (Figure 6). We found that elliptical area was minimum with no load and it increased by $40 \%$ with posterior load carriage and by about $130 \%$ on HLC. There was no significant difference found for COP path length at the level and uphill condition whereas downhill condition had significantly higher path length ( $17 \%$ more) in contrast to the other conditions. It was also seen that path length was also minimum with no load but it increased by $18 \%$ with PLC and by $57 \%$ with HLC. We found that downhill condition had significantly higher COP sway velocity. Also, HLC condition had significantly higher COP velocity than uphill and level surface standing condition. Steadiness perceptive rating (SPR) for standing on a flat surface was found to be highest with a mean of 9.8 units (Figure 7), and these ratings decreased to 7.9 and 7.0 for PLC and HLC respectively. Standing on the downhill was found to be slightly more challenging by subjects than uphill standing with scores of 8.7 versus 8.8 units. Subjects also found HLC condition more challenging for maintaining balance than PLC with a mean score of 6.25 and 7.1 units (Figure 7). 
It was found that multiscale entropy values with HLC was significantly lower than that of PLC and no load condition $(p<0.0001)$ (Figure $8 a$ and $b)$. Similar results were found for approximate entropy and sample entropy values $(p<0.001)$. We also found that there was a significant increase in persistence in the COP time series in PLC $(p<0.0001)$ and OLC $(p<0.0001)$ load carriage conditions (Figure $8 c)$. It was found that multiscale entropy, ApEn, and SampEn values were significantly lower while standing on a flat surface than that on Inclined surfaces $(p<0.0001)$ (Figure 10a and $b$ ). We also found that there was a significant decrease in the persistence of COP signals with the inclination $(p<0.0001)$ with downhill showing the highest antipersistence (Figure 10c). It was found that increase in load magnitude led to significant decline in complexity as evidenced by MSE, ApEn, and SampEn $(p<0.001)$ but significant increase in the persistence of COP signals $(p<0.001)$ (Figure 9).

\section{Discussion}

The aim of this study was to examine changes in postural control through postural parameters while standing on different SI with different LM and LC method. Our primary hypothesis supports our findings. Results suggest that postural instability related fall risk increased with standing on inclined surfaces and are influenced by the LC method. Accordingly, in the present article, we found that SI and LC methods augment to postural instability. HLC and uphill or downhill facing conditions equally fostered balance problems. Furthermore, this study underlined the importance of quantifying non-linear variability for understanding the biomechanics of inclined standing posture.

We found that there is a serious compromise of balance when carrying load and exposure to the inclined surface, this may further decrease tolerance of perturbation before fall occurs; the risk of loss of balance and associated injury may be greater(Chow, Kwok et al. 2006). Postural stability is therefore, important for the prevention of pain and injuries related to loss of balance, especially when performing complicated on inclined surfaces and carrying loads. Inclination, load-bearing may impair complexity (as measured by the entropy of COP signals) of a system by affecting the readiness of the neuromuscular control system (mark of alertness) for the unexpected by becoming rigid in its control(Borg and Laxaback 2010). An impaired neuromuscular control may become rigid and become unable to successfully cope with new challenges to the maintenance of balance. Figure 4 shows the stabilographic recording of one of the subjects at the inclined uphill, inclined downhill and flat surface. We found that sway recordings are most dense at the uphill surface, followed by a downhill surface and least dense at a flat surface. We found that the sway during no-load condition at all the three surfaces is having least sway as compared to load carrying conditions at these surfaces. This increased sway may reflect both more intensive controls to maintain postural stability and changes in movement strategies during exposure to these challenging conditions i.e... inclined surface and load carriage. 
Apparently, the postural sway/range from center of pressure in both mediolateral and anteroposterior directions was found to be considerably affected by SI and COP shifted extremely towards toes in downhill standing and similarly towards heels in uphill standing, and thus SI influenced COP area to a large extent. We found that elliptical COP area was substantially different from the level surface compared against the values obtained on inclined surfaces. The level surface had significantly higher elliptical COP area than both downhill and uphill conditions. It was maximum on a level surface, and it decreased at the inclined surface, i.e. $25 \%$ and $32 \%$ at downhill and uphill condition respectively. We also found that elliptical area was significantly different for two kinds of load carriage methods; minimum with no load and it increased by $40 \%$ with posterior load carriage and by about $130 \%$ on head load carriage. Thus, signifying head load carriage inducing imbalance among participants.

Emerich, et. al. indicated that balance was significantly affected by exposure to varying degrees of inclination (Emerich). Similar results were obtained by other researchers, who studied postural sway on sloped surfaces (Simeonov, Hsiao et al. 2003). They found that increase of surface slope resulted in a progressive increase of postural sway in the A-P direction, and body sways occurred predominantly in the A-P direction as well. They also mentioned that slope reduced the effective base of support (i.e. the area of the footprint projection on a horizontal plane) that limited the safe range of movement of the body's center of gravity and thus increased postural instability. Both LC and LM increased mediolateral (COPy) and anterior-posterior directions (COPx) in appreciable amount and indeed at the inclined surface (uphill and downhill) as compared to that at the level surface.

Previous research (Pai and Patton 1997, Wade, Weimar et al. 2004) had reported the whole-body COP velocity as an important factor in predicting balance conditions of the participants. It is a measure of the angular change of the COP per unit time, where the value is representative of changes to the location of the COP in the anterior, posterior, medial, and/or lateral directions. Higher values indicate decreased postural stability, as they imply larger angular changes with the location of the COP. There were no significant differences found for COP velocity on the level and uphill conditions but downhill condition had significantly higher COP velocity from the other two conditions. We also found that head load carriage condition had significantly higher COP velocity than uphill and level surface standing condition. Since postural sway represents an effective indicator of balance abilities during standing on leveled/inclined surfaces with different LM and LC methods, the alterations observed to suggest that SI, LC, and LM lead to balance impairment. This increased sway velocity of COP at a downhill inclined surface reflects higher postural instability as well. Also, the increased postural sway velocity at sloped surfaces may reflect both more intensive balance control and postural exploration during instability in a phase of postural adaptation to these challenging conditions. There was no significant difference found for COP path length on the level and uphill condition whereas downhill condition had significantly higher path length (17\% more) in contrast to the other conditions. It was also seen that path length was also minimum with no load, but it increased by $18 \%$ with posterior load carriage and by $57 \%$ on the head load carriage.

As standing on inclined planes is an abnormal condition of standing, it places increased gravitoinertial demands on the head during standing. The increased 
gravitoinertial demands indicate an alteration in otolith information during incline standing as compared with level standing. While ascending the incline, increased neck and trunk flexion compound anterior displacement of the head's center of mass to further increase gravitoinertial requirements for head stabilization in this condition. These finding regarding increased trunk flexion orientation while ascending the inclined plane has also been previously reported(Cromwell 2003).

According to motor control theory, one of the main aspects of motor control is to orient the body (involving trunk, head, neck, shoulders, etc.) to maintain a posture to minimize the deterioration of balance, thus stabilizing the whole-body center of gravity to prevent a fall. PLC is symmetrical carrying on both shoulders, whereas HLC may cause asymmetric and compromised head movements leading to reduced peripheral vision and altered postural stability. Therefore, with HLC or PLC, the subjects try to stabilize their body-load system center of gravity. This is usually achieved by trunk forward inclination(Bloom and Woodhull-Mcneal 1987). Similarly, in this study we found that when standing or carrying the load on an inclined surface, there is an increase in forward inclination of the trunk. Locating the load as close as possible to the body center of gravity (posterior vs head) may result in lower energy cost but (Knapik, Harman et al. 1996), because subjects leaned due to SI inclination and LC method (Hong and Li 2005, Smith, Ashton et al. 2006), this may lead to the development of spinal pain. PLC is also associated with shifting the trunk forward for counterbalancing load at their back, and this is well in agreement with findings of load carriage studies on adults (Martin and Nelson 1986, Kinoshita 2007) and in children (Pascoe, Pascoe et al. 1997, Chansirinukor, Wilson et al. 2001).

Subjective measures such as steadiness perceptive rating can reflect the status or quality of human postural control, and we found that perceived steadiness corresponds well with objective measures of postural control. In this study, we found a general consistency between perceived steadiness and the COP-based measures with respect to the influences of SI, LC, and LM. (Figure 7). Descriptive statistics such as COP area, velocity and ranges could conceal the control principles that underlie the observed postural dynamics, thus this study also looks into quantifying the variability of postural control strategies in standing on inclined surfaces with two carrying strategies and load magnitudes. Linear descriptors of variability such as standard deviations and/or coefficient of variation are complemented with non-linear measures of variability such as approximate entropies, sample entropy, Hurst exponent and multiscale entropies(Stergiou 2004). Such variability analysis tools can quantify subtle changes in the dynamics of biological systems(Lipsitz and Goldberger 1992).

We investigated how inclination affected sensory signals, which further affected the temporal structure of sway variability. The findings also demonstrate that standing on inclined planes increased postural instability. We attempted to expand the relation between COP variability measures with different postural challenging conditions, where a different amount of attention invested with compromising of balance. Postural control is one of highly automatic activity with limited attention investments, and these attention investments increase with postural challenges or with impaired sensory information (Roerdink, Hlavackova et al. 2011). In a previous 
study, it has been found that standing has more attention demands than standing (Lajoie, Teasdale et al. 1993, Teasdale, Bard et al. 1993, Lajoie, Teasdale et al. 1996, Vuillerme, Forestier et al. 2002, Vuillerme and Nougier 2004, Vuillerme, Isableu et al. 2006) and thus COP fluctuations were more regular for standing than sitting (Roerdink, Hlavackova et al. 2011). Since standing on an inclined plane increases postural challenges and attention demands, we expected that regularity of these postural signals would increase with a challenge to postural control. Our results corroborated by studies relating COP regularity and amount of attention invested according to balance demands (Donker, Roerdink et al. 2007, Donker, Ledebt et al. 2008, Stins, Michielsen et al. 2009).

In the present study, the relation between distinct imposed balance demands and complexity measures corroborated by our results. Thus, balance is evaluated under the different base of support configurations and load carriage by examining nonlinear variability measures such as sway complexity.

Limitation: While this study provided insight into the load carriage at sloped standing, there were still several limitations present. First, all the data presented are only valid for the slope angle used in the study, which was selected as 20 degrees for this study. Secondly, fall risk or propensity for loss of balance have been measured indirectly, but postural control was instead evaluated under controlled static conditions. However, several of the COP-based measured used here have been demonstrated as valid indices of the risk of falling (Fernie, Gryfe et al. 1982, Lichtenstein, Shields et al. 1988, Maki, Holliday et al. 1990). It is also limited, to some extent, since postural control was measured with a constrained stance (standing with feet together), which is not typical in occupational settings or daily life. Stance width has been demonstrated to influence postural control, with a wider stance leading to less postural sway(Kirby, Price et al. 1987). We conducted this study in US population, who is unlikely to have any experience in HLC. This unfamiliarity of tasks could probably have highlighted postural instability among this study population.

\section{Conclusion}

This study could identify risky conditions for falls when presented with different SI, LC, and LM. The most important conclusive implication of this study is the limiting load in HLC especially on the inclined surface as it leads to more unstable posture and increased instability. In the present study, we found that the downhill standing increases the fall risk substantially even when subjects were carrying loads from that of flat surface standing. It is suggestive that the aim of adjusting posture under conditions of load carriage is to minimize energy expenditure. Since in PLC load was closer to body COM than in HLC, therefore, it was not surprising to find HLC as more risk-prone to fall than PLC in this study. Future studies need to be conducted to assess threshold/ carry limit normalized per body weight for safe HLC. Here both uphill and downhill inclinations induced postural instability, though the downhill condition is a more unstable condition for participants to maintain balance than that at uphill and level surface conditions. Given the increasingly detrimental effect of inclination angle on postural control, working surfaces should be horizontal wherever possible, or minimized otherwise. Further impending studies can also look into safe load carriage method as per workplace inclination. As injuries associated with load carriage involve increased risk of falls; therefore, educating workers on carrying 
strategy closer to the center of mass is likely to prevent prospective injury by load carriage on inclines.

\section{Summary}

- We found that i) load carriage method, ii) surface inclination, and iii) load magnitude all contributed to variability in postural sway when compared to control at level surface condition.

- Both linear and nonlinear variabilities are crucial in understanding postural instability and risk of fall or injuries.

- Load carriage method influenced postural stability, especially head load carriage considerably induced postural instability and reduced complexity of postural sway signals. This loss of complexity may result in loss of adaptability during perturbation.

Acknowledgements

The authors highly appreciate contributions of Prof. Maury A. Nussbaum (H.G. Prillaman Professor) at Grado Department of Industrial and Systems Engineering, Virginia Tech. This research work was not possible without his guidance and help. He served as Hema's advisor during her Fullbright Foreign Student Program, during which the data were collected for this study. 

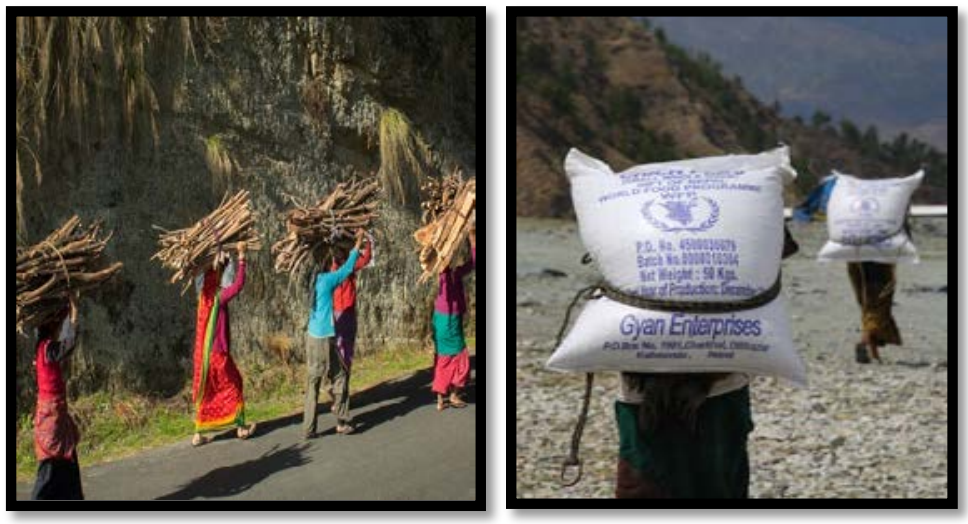

Figure 1. Head and posterior load carriage practice among agricultural workers
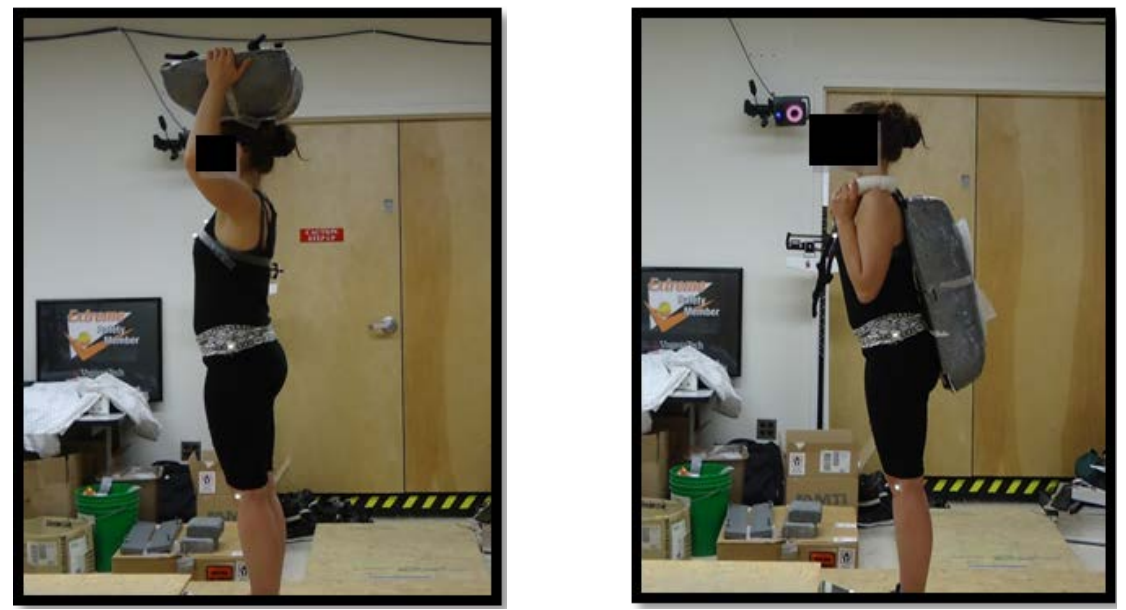

Figure 2. Methods of load carriage: posterior load carriage (left), and head load carriage (right) 


\section{(a) PERCEPTION OF STEADINESS}
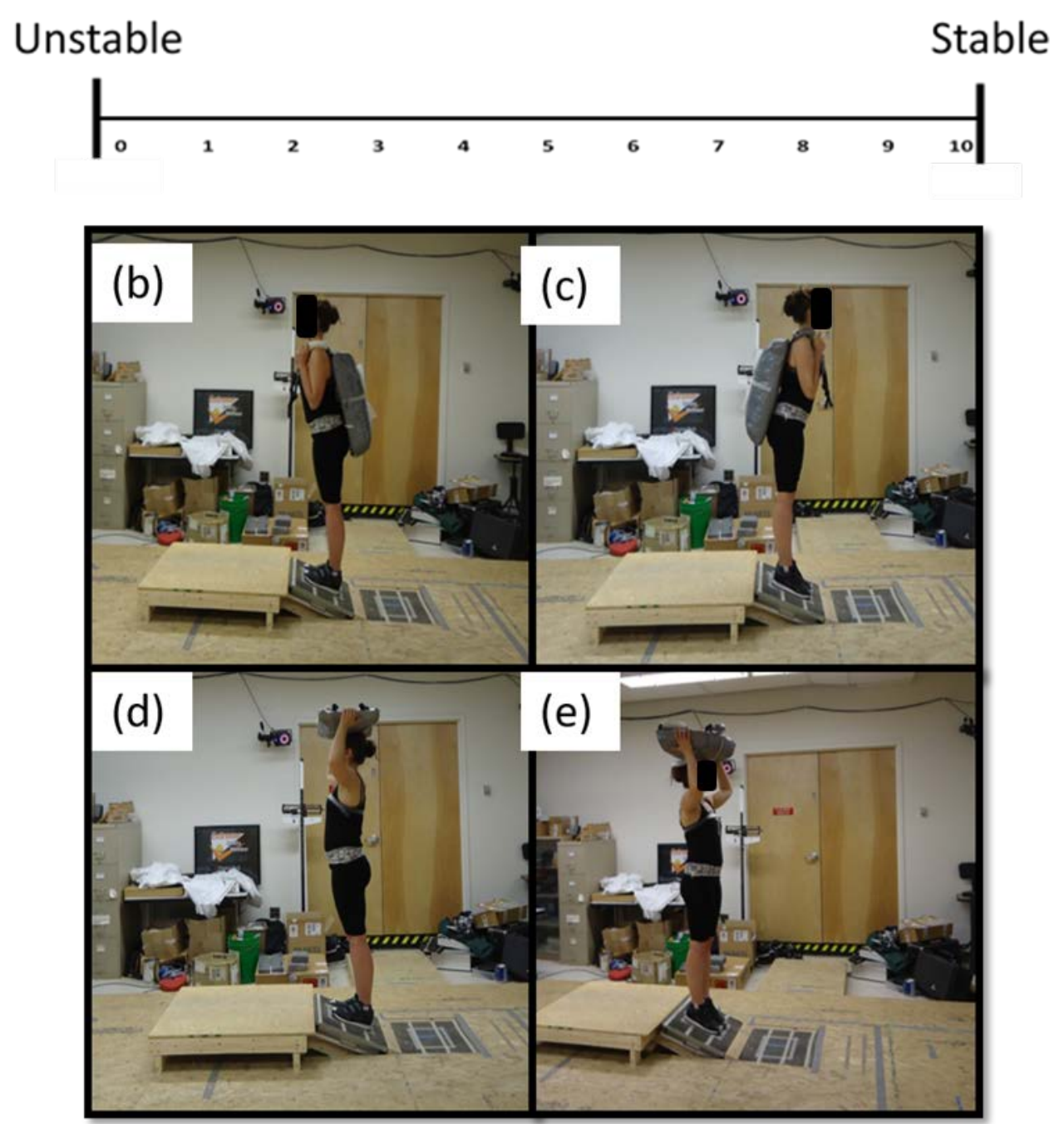

Figure 3. a) Perception of steadiness subjective scale. Experimental setup, with uphill (b and d) and downhill (c and e) positions, while participant carried the load on the back (PLC) (b and c) and head (HLC) (d and e). 
(a)

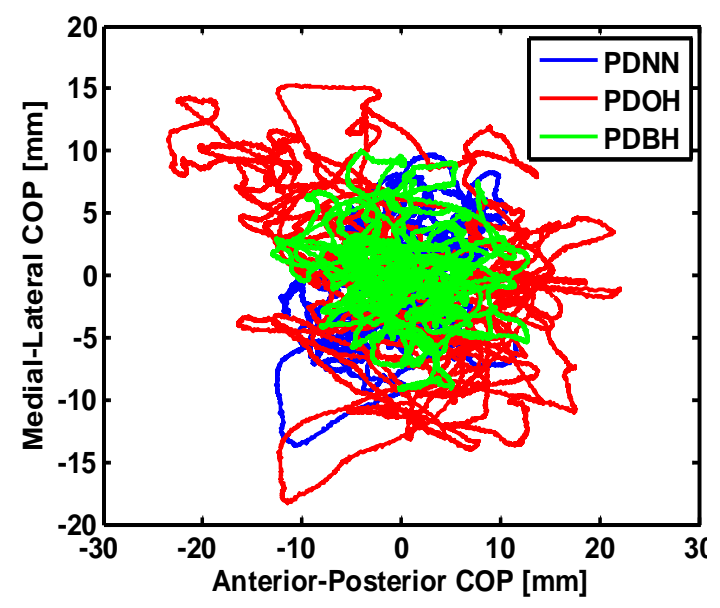

(b)

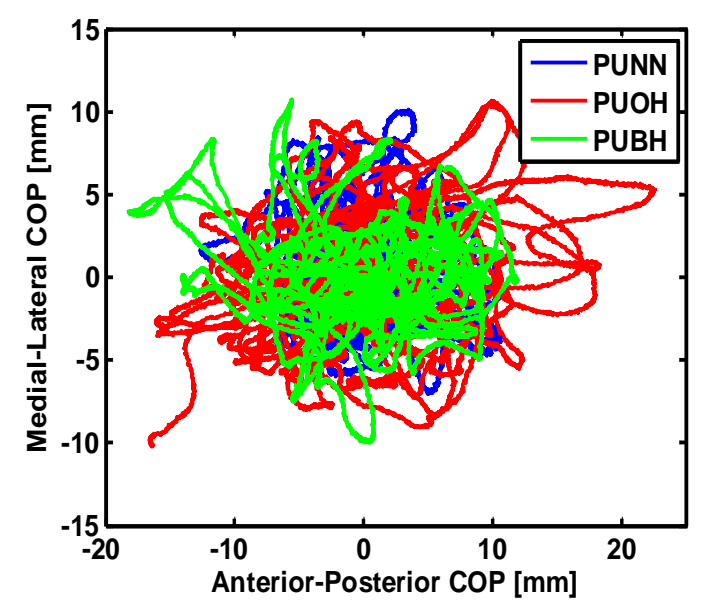

(c)

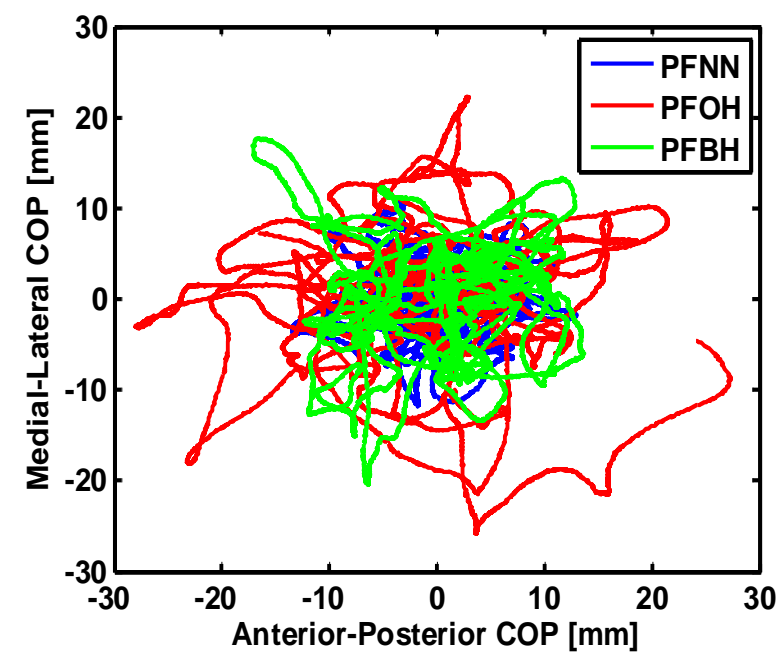

Figure 4 : Stabilographic recordings of a representative normal, subject during quiet stance at downhill surface with no load( PDNN), heavy load on head( $\mathrm{PDOH})$, and heavy load on back(PDBH), at uphill surface with no load( PUNN), heavy load on head( PUOH), and heavy load on back(PUBH), at flat surface. with no load( PFNN), heavy load on head( PFOH), and heavy load on back(PFBH) 
(a)

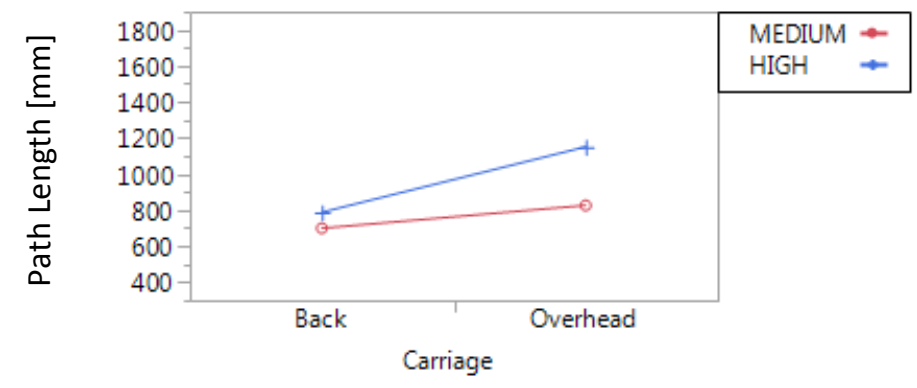

(b)

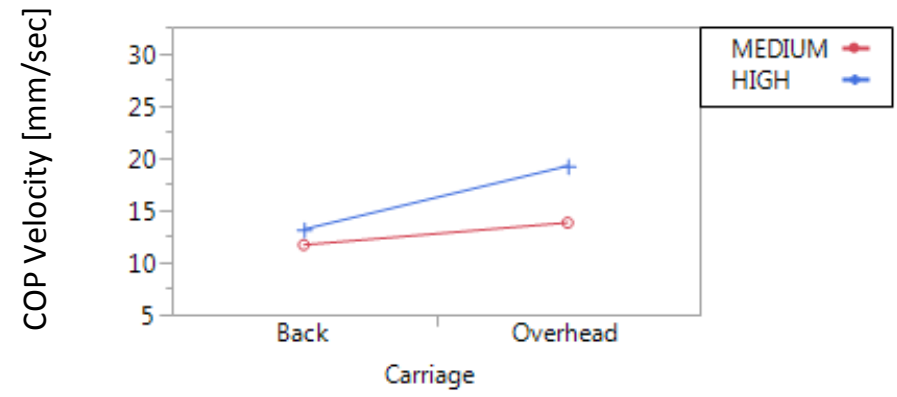

(c)

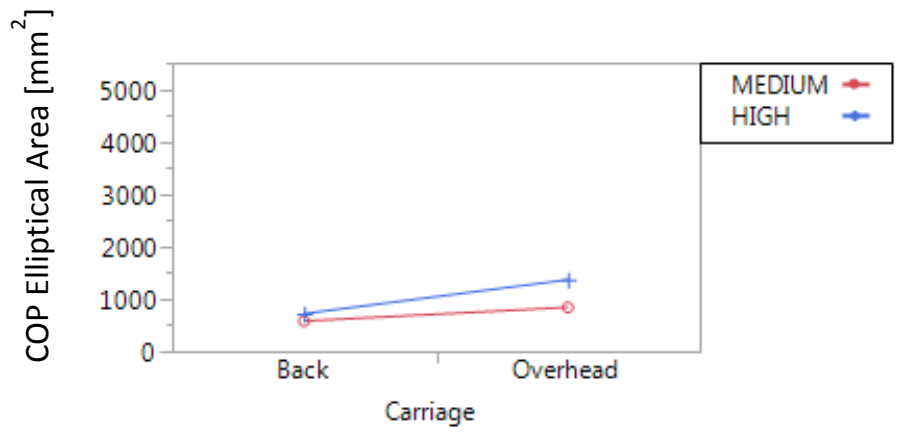

Figure 5: Interaction effects among independent variables magnitude of load and strategy of load carriage for dependent variables a) path length, b) COP Velocity and c) COP Elliptical A 
(a)

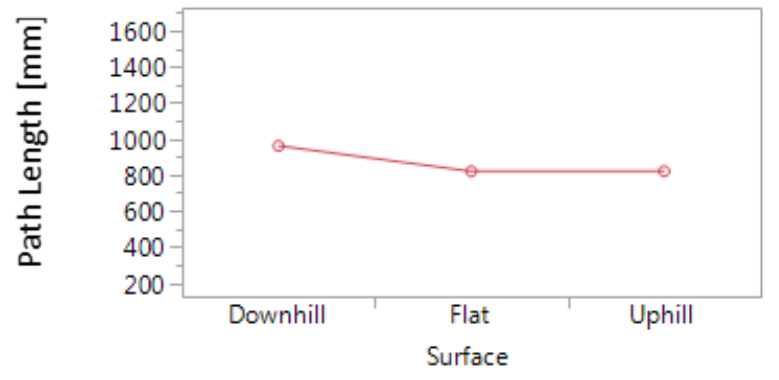

(b)

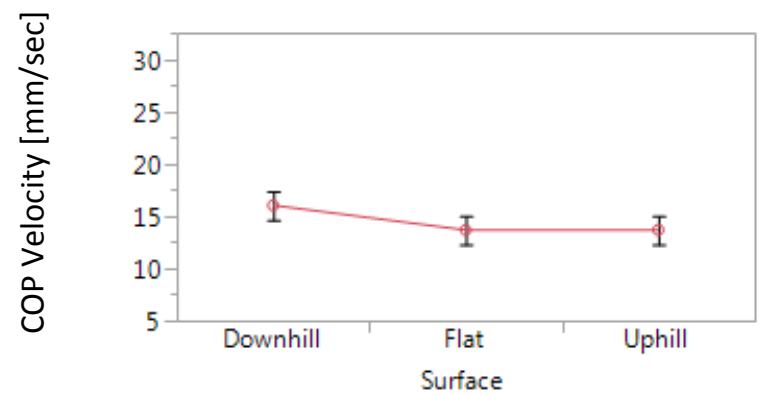

(c)

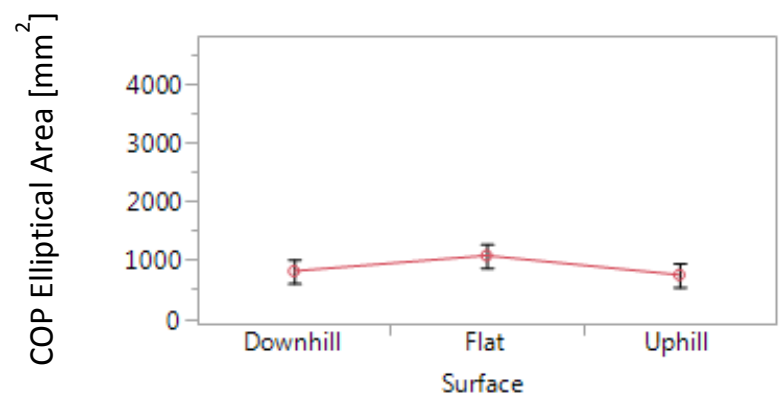

Figure 6 : Effects of surface inclinations on dependent variables a) path length, b) COP Velocity and c) COP Elliptical 
Table 1: Summary of ANOVA results ( $p$ values) for main and interactive effects of SI, LC, and LM.

\begin{tabular}{lllllll} 
& SI & LC & LM & SI×LC & SI×LM & LC $\times$ LM \\
\hline Range COPx & $<\mathbf{0 . 0 0 0 1}$ & $<\mathbf{0 . 0 0 0 1}$ & $\mathbf{0 . 0 0 0 2}$ & $\mathbf{0 . 0 2 6 1}$ & $\mathbf{0 . 0 1 6 4}$ & $<\mathbf{0 . 0 0 0 1}$ \\
\hline Range COPy & $<\mathbf{0 . 0 0 0 1}$ & $<\mathbf{0 . 0 0 0 1}$ & $<\mathbf{0 . 0 0 0 1}$ & $<0.0001$ & $\mathbf{0 . 0 3 2}$ & $\mathbf{0 . 0 1 4 4}$ \\
\hline Circular Area & $<\mathbf{0 . 0 0 0 1}$ & $<\mathbf{0 . 0 0 0 1}$ & 0.6116 & $<0.0001$ & $\mathbf{0 . 0 0 0 7}$ & 0.1573 \\
\hline COP Path Length & $<\mathbf{0 . 0 0 0 1}$ & $<\mathbf{0 . 0 0 0 1}$ & $<\mathbf{0 . 0 0 0 1}$ & 0.9272 & 0.5468 & $<\mathbf{0 . 0 0 0 1}$ \\
\hline COP Velocity & $<\mathbf{0 . 0 0 0 1}$ & $<\mathbf{0 . 0 0 0 1}$ & $<\mathbf{0 . 0 0 0 1}$ & 0.9274 & 0.547 & $<0.0001$ \\
\hline Elliptical Area & $\mathbf{0 . 0 0 0 3}$ & $<\mathbf{0 . 0 0 0 1}$ & $\mathbf{0 . 0 0 0 1}$ & 0.221 & 0.1562 & $<0.0001$ \\
\hline
\end{tabular}

Table 2: This table shows mean and standard deviation of Center of pressure parameters at various magnitude of load, load carriage style and inclinations.

\begin{tabular}{|c|c|c|c|c|c|c|}
\hline \multirow{2}{*}{\multicolumn{2}{|c|}{$\begin{array}{c}\text { Load Magnitude } \\
\text { Load Carriage Strategy }\end{array}$}} & \multirow{3}{*}{$\begin{array}{c}\text { LOW } \\
\text { Control } \\
\text { Mean } \pm \text { SD }\end{array}$} & \multicolumn{2}{|c|}{ MEDIUM } & \multicolumn{2}{|c|}{ HIGH } \\
\hline & & & \multirow{2}{*}{$\begin{array}{r}\text { Back } \\
\text { Mean } \pm \text { SD }\end{array}$} & \multirow{2}{*}{$\begin{array}{r}\text { Overhead } \\
\text { Mean } \pm \text { SD }\end{array}$} & \multirow{2}{*}{$\begin{array}{r}\text { Back } \\
\text { Mean } \pm \text { SD }\end{array}$} & \multirow{2}{*}{$\begin{array}{r}\text { Overhead } \\
\text { Mean } \pm \text { SD }\end{array}$} \\
\hline Surface & & & & & & \\
\hline \multirow[t]{6}{*}{ Downhill } & Range_COPX & $33.64 \pm 13.68$ & $38.07 \pm 12.18$ & $45.02 \pm 14.51$ & $43.42 \pm 14.98$ & $63.40 \pm 21.19$ \\
\hline & Range_COPY & $19.13 \pm 6.26$ & $20.46 \pm 5.36$ & $24.75 \pm 6.92$ & $24.26 \pm 6.65$ & $31.24 \pm 7.07$ \\
\hline & Circular_Area & $885.36 \pm 1612.53$ & $962.54 \pm 899.80$ & $1331.78 \pm 1137.31$ & $1170.36 \pm 1714.85$ & $1867.11 \pm 1354.44$ \\
\hline & PathLength & $719.44 \pm 201.28$ & $785.36 \pm 197.11$ & $909.67 \pm 211.60$ & $883.23 \pm 198.23$ & $1265.27 \pm 315.89$ \\
\hline & COP_Velocity & $11.99 \pm 3.35$ & $13.09 \pm 3.29$ & $15.16 \pm 3.53$ & $14.72 \pm 3.30$ & $21.09 \pm 5.26$ \\
\hline & Elliptical_Area & $433.96 \pm 346.29$ & $481.16 \pm 266.34$ & $769.61 \pm 547.96$ & $670.97 \pm 492.32$ & $1317.48 \pm 729.51$ \\
\hline \multirow[t]{6}{*}{ Flat } & Range_COPX & $26.78 \pm 6.17$ & $31.75 \pm 8.58$ & $32.76 \pm 7.68$ & $32.01 \pm 8.67$ & $42.15 \pm 12.81$ \\
\hline & Range_COPY & $31.43 \pm 12.15$ & $31.59 \pm 9.50$ & $40.54 \pm 15.78$ & $36.48 \pm 11.18$ & $56.86 \pm 21.15$ \\
\hline & Circular_Area & $9380.80 \pm 4221.35$ & $13014.28 \pm 5713.87$ & $9220.72 \pm 3596.68$ & $11900.84 \pm 4566.46$ & $6463.97 \pm 3171.88$ \\
\hline & PathLength & $606.02 \pm 146.89$ & $661.86 \pm 182.46$ & $793.04 \pm 170.88$ & $739.30 \pm 146.02$ & $1085.58 \pm 299.50$ \\
\hline & COP_Velocity & $10.10 \pm 2.45$ & $11.03 \pm 3.04$ & $13.22 \pm 2.85$ & $12.32 \pm 2.43$ & $18.09 \pm 4.99$ \\
\hline & Elliptical_Area & $600.35 \pm 349.43$ & $733.64 \pm 442.51$ & $992.10 \pm 658.89$ & $870.39 \pm 567.18$ & $1701.52 \pm 1083.00$ \\
\hline \multirow[t]{2}{*}{ Uphill } & Range_COPX & $33.20 \pm 10.57$ & $41.71 \pm 15.83$ & $48.44 \pm 13.51$ & $42.60 \pm 13.62$ & $56.19 \pm 20.10$ \\
\hline & Range_COPY & $15.66 \pm 3.67$ & $18.99 \pm 6.04$ & $23.42 \pm 7.03$ & $23.61 \pm 9.92$ & $28.64 \pm 7.40$ \\
\hline
\end{tabular}




\begin{tabular}{|l|l|l|l|l|l|l|}
\hline & Circular_Area & $7305.08 \pm 4663.04$ & $8058.57 \pm 5105.02$ & $6979.23 \pm 4582.88$ & $9780.09 \pm 6250.83$ & $7275.89 \pm 4687.43$ \\
\hline & PathLength & $558.01 \pm 136.39$ & $652.77 \pm 163.96$ & $778.25 \pm 186.53$ & $744.55 \pm 169.06$ & $1105.30 \pm 319.26$ \\
\hline & COP_Velocity & $9.30 \pm 2.27$ & $10.88 \pm 2.73$ & $12.97 \pm 3.11$ & $12.41 \pm 2.82$ & $18.42 \pm 5.32$ \\
\hline & Elliptical_Area & $365.57 \pm 180.34$ & $522.70 \pm 282.47$ & $756.63 \pm 396.87$ & $619.75 \pm 303.46$ & $1081.96 \pm 580.85$ \\
\hline
\end{tabular}

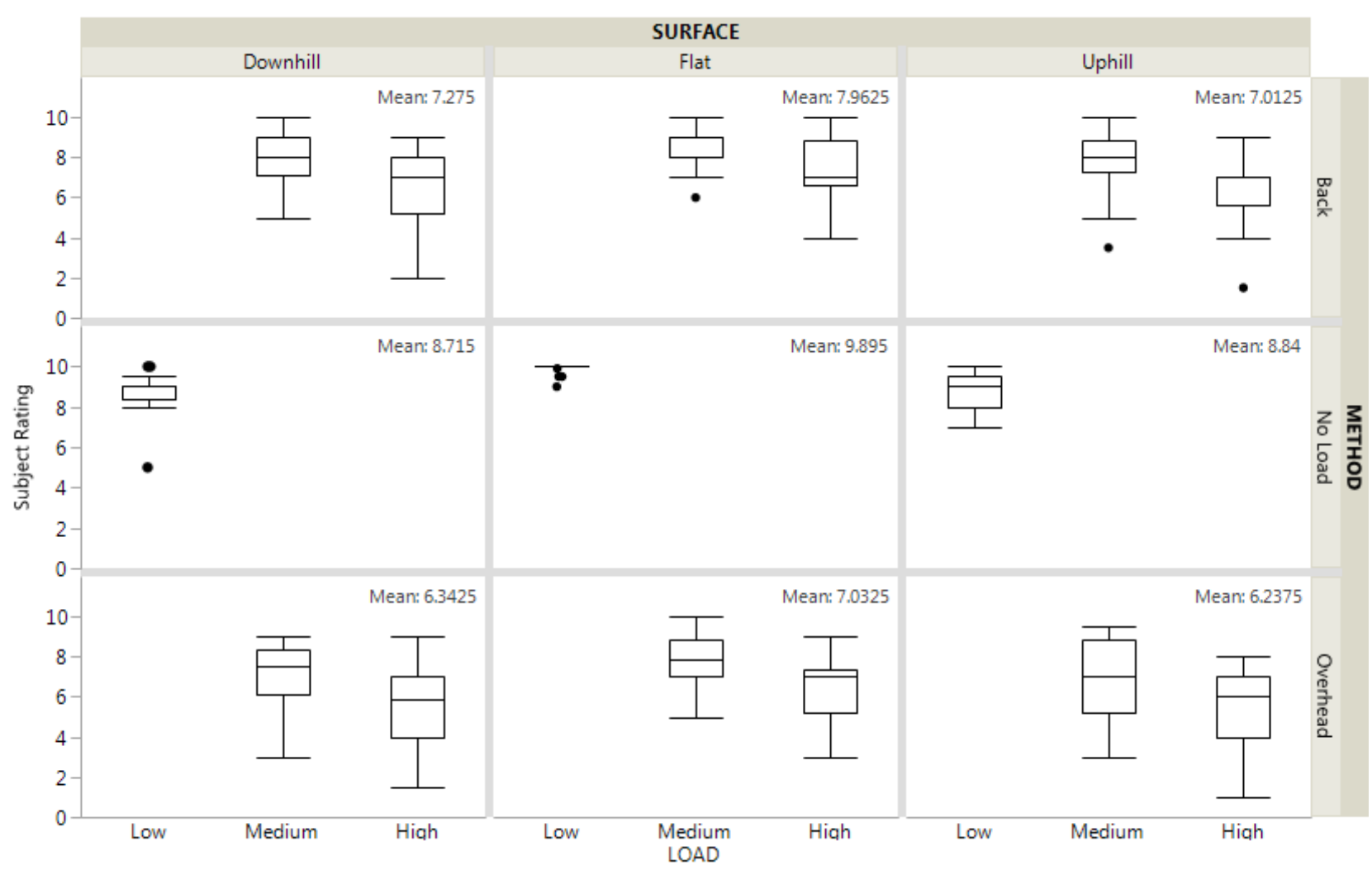

Figure 7: Subjective rating of perceived stability. 


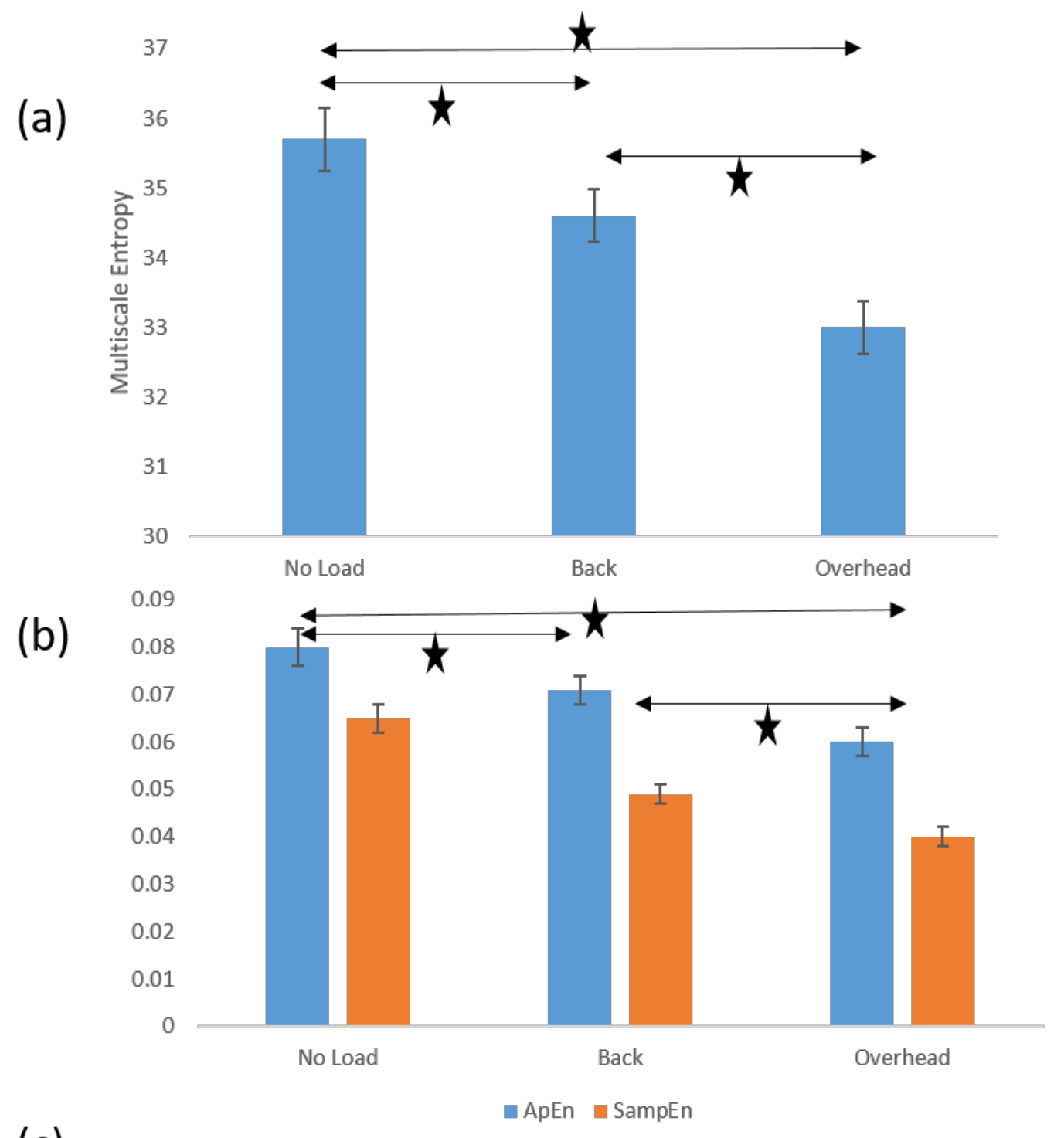

(c)

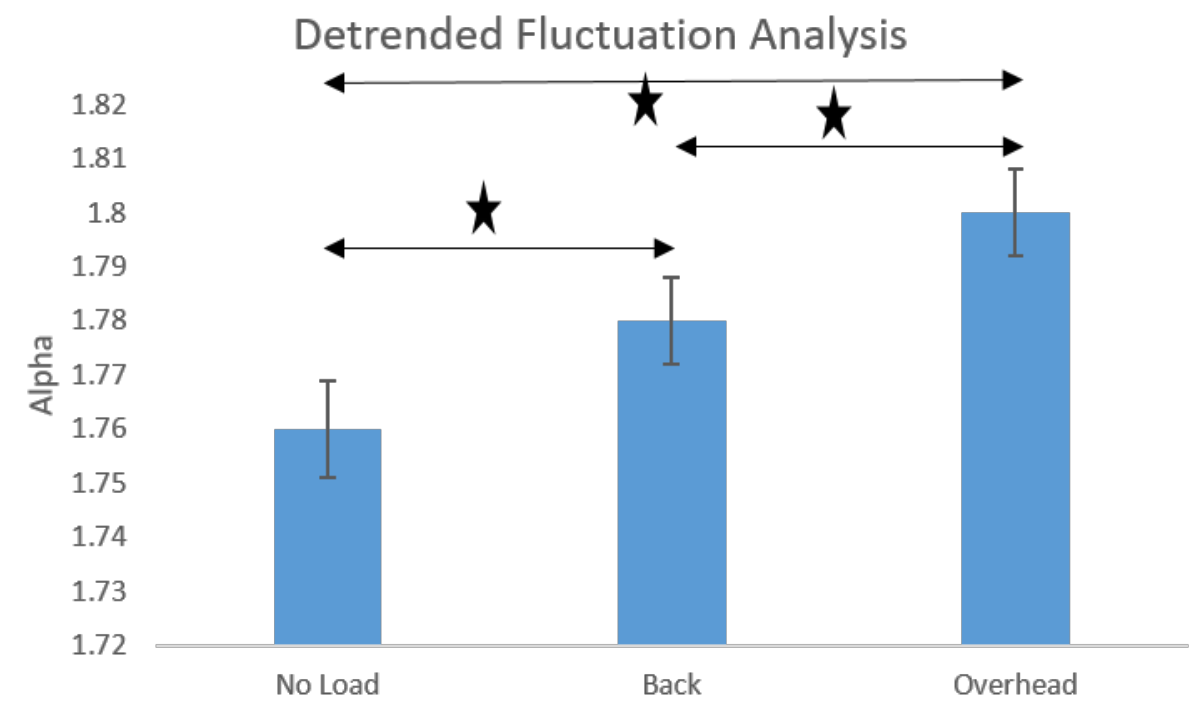

Figure 8: a) Multiscale entropy, b) Approximate entropy (ApEN) and Sample Entropies (SampEn) c) hurst exponent (alpha) values with different methods of load carriages. 


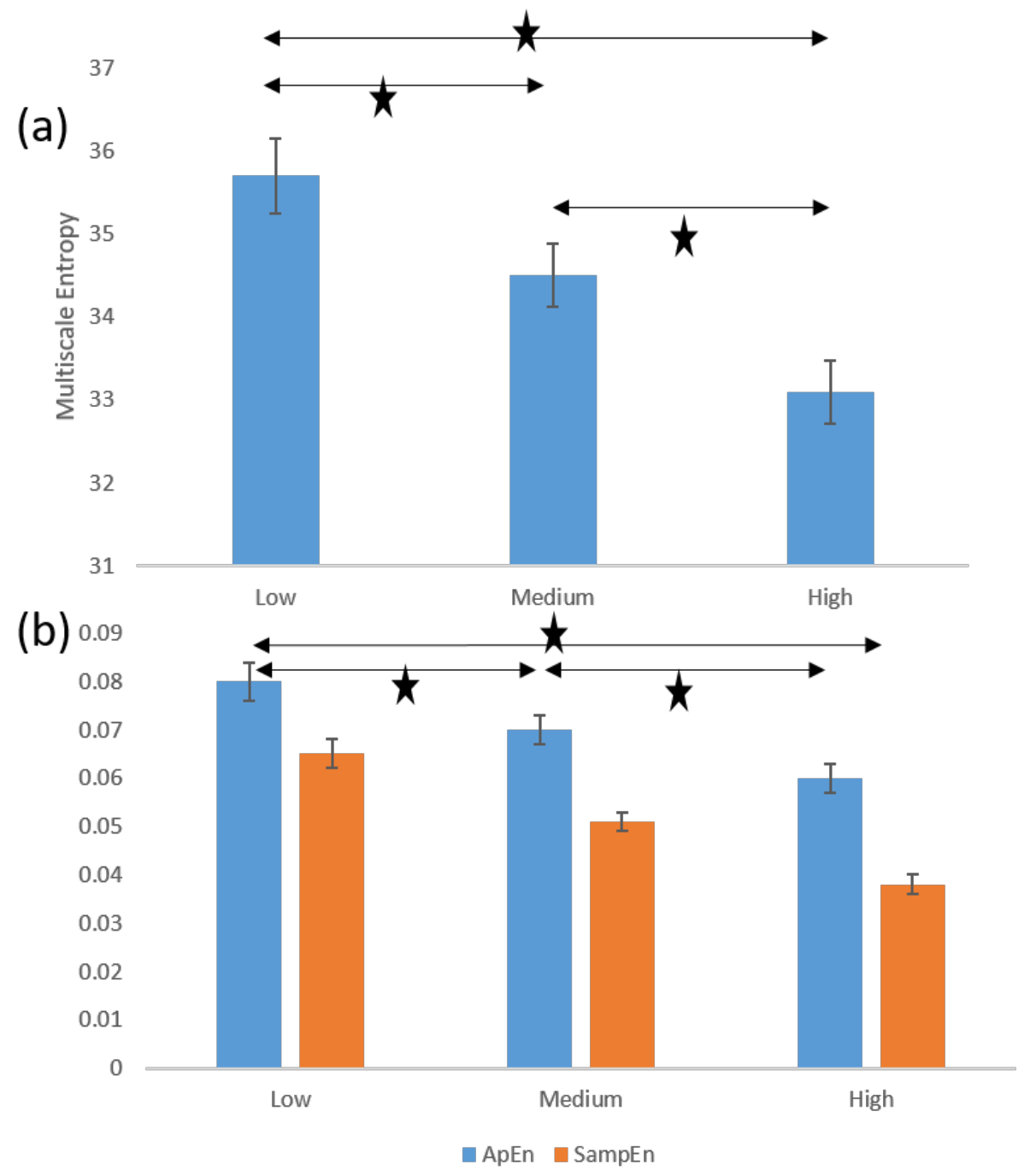

(c)

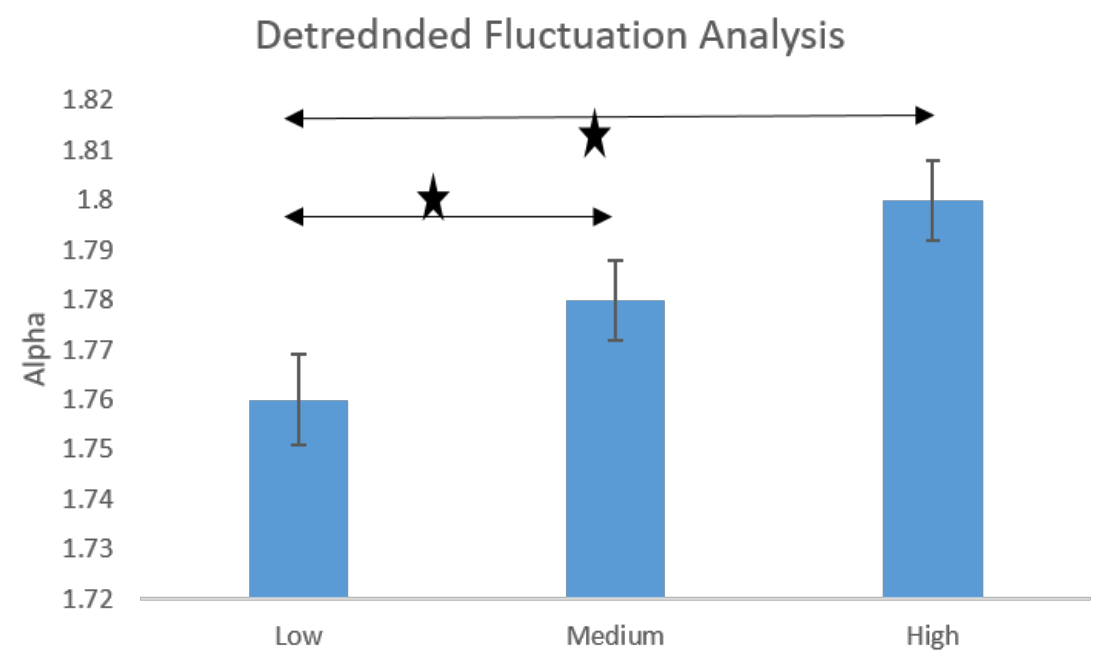

Figure 9: a) Multiscale entropy, b) Approximate entropy (ApEN) and Sample Entropies (SampEn) c) hurst exponent (alpha) values with different magnitudes of loads. 


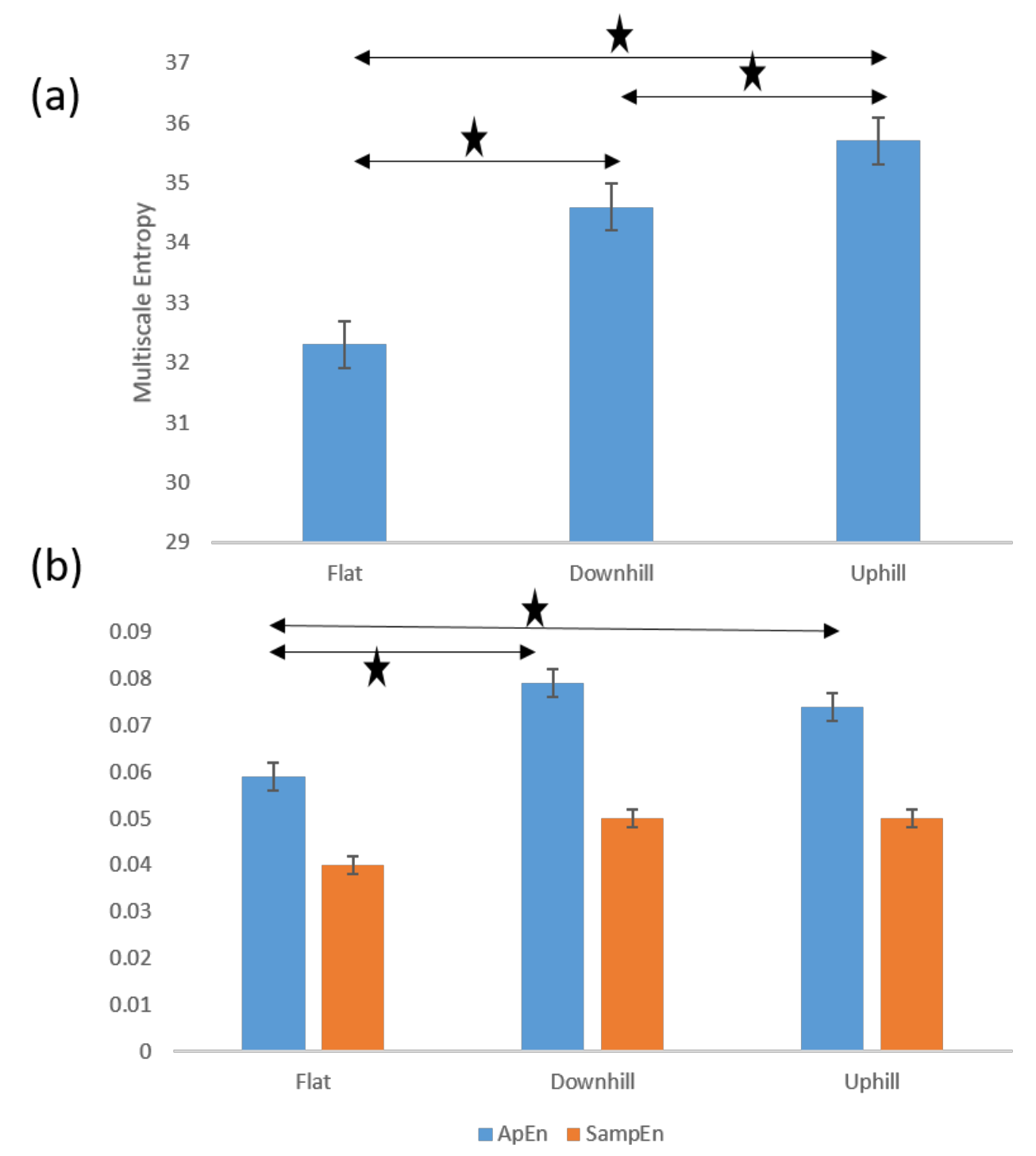

(c)

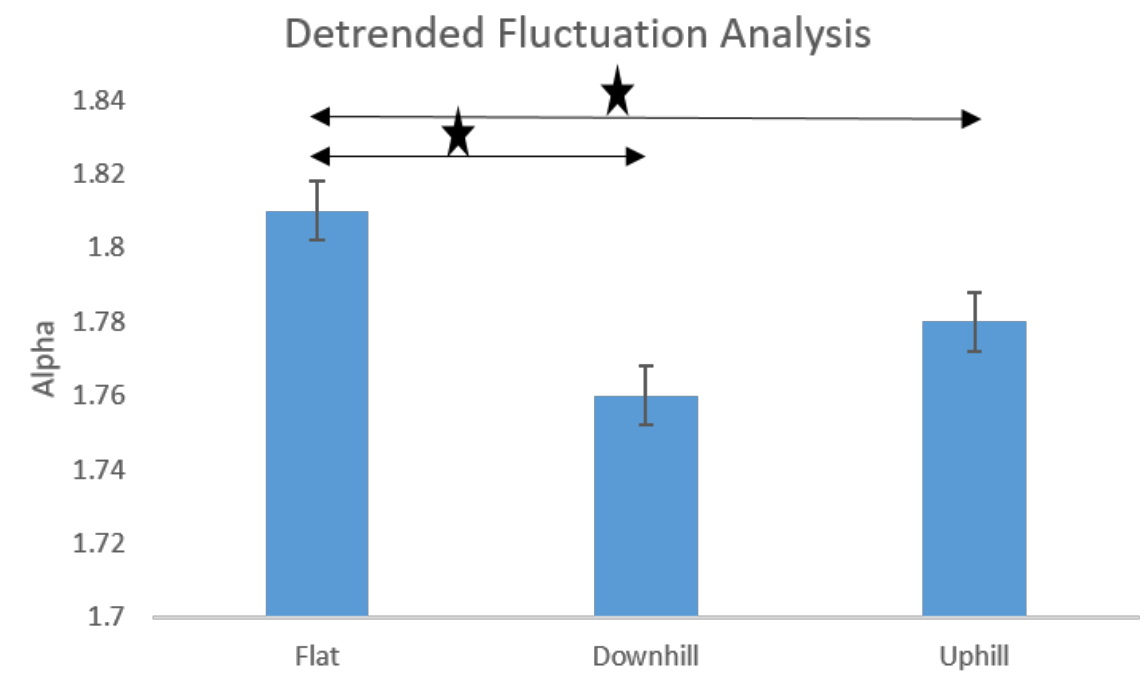

Figure 10: a) Multiscale entropy, b) Approximate entropy (ApEN) and Sample Entropies (SampEn) c) hurst exponent (alpha) values with different surface inclinations. 
Table 3: This table shows mean and standard deviation of non-linear parameters of Center of pressure at various magnitude of load, load carriage style and inclinations.

\begin{tabular}{|c|c|c|c|c|c|c|c|c|c|c|}
\hline & & & \multicolumn{2}{|c|}{ MSE } & \multicolumn{2}{|c|}{ ApEn } & \multicolumn{2}{|c|}{ SampEn } & \multicolumn{2}{|c|}{ DFA } \\
\hline & & & \multicolumn{2}{|c|}{ Direction } & \multicolumn{2}{|c|}{ Direction } & \multicolumn{2}{|c|}{ Direction } & \multicolumn{2}{|c|}{ Direction } \\
\hline & & & AP & ML & AP & ML & AP & ML & AP & ML \\
\hline $\begin{array}{l}\text { Inclinati } \\
\text { on }\end{array}$ & Carriage & Load & Mean \pm SD & Mean \pm SD & Mean \pm SD & Mean \pm SD & Mean \pm SD & Mean \pm SD & Mean \pm SD & Mean \pm SD \\
\hline \multirow[t]{5}{*}{ Downhill } & Back & Medium & $32.14 \pm 3.53$ & $37.97 \pm 3.36$ & $0.07 \pm 0.02$ & $0.08 \pm 0.05$ & $0.04 \pm 0.01$ & $0.07 \pm 0.03$ & $1.75 \pm 0.06$ & $1.73 \pm 0.06$ \\
\hline & & High & $32.57 \pm 2.52$ & $37.53 \pm 3.30$ & $0.06 \pm 0.02$ & $0.09 \pm 0.06$ & $0.03 \pm 0.01$ & $0.06 \pm 0.04$ & $1.79 \pm 0.07$ & $1.75 \pm 0.07$ \\
\hline & NoLoad & Low & $33.53 \pm 2.97$ & $38.42 \pm 4.14$ & $0.08 \pm 0.03$ & $0.12 \pm 0.06$ & $0.04 \pm 0.01$ & $0.09 \pm 0.03$ & $1.74 \pm 0.08$ & $1.71 \pm 0.09$ \\
\hline & Overhead & Medium & $32.04 \pm 3.41$ & $37.02 \pm 2.64$ & $0.06 \pm 0.02$ & $0.08 \pm 0.04$ & $0.02 \pm 0.01$ & $0.06 \pm 0.03$ & $1.81 \pm 0.06$ & $1.78 \pm 0.08$ \\
\hline & & High & $29.72 \pm 2.71$ & $35.54 \pm 3.50$ & $0.05 \pm 0.02$ & $0.07 \pm 0.04$ & $0.02 \pm 0.00$ & $0.04 \pm 0.03$ & $1.80 \pm 0.06$ & $1.78 \pm 0.06$ \\
\hline \multirow[t]{5}{*}{ Flat } & Back & Medium & $33.82 \pm 3.61$ & $33.31 \pm 2.49$ & $0.06 \pm 0.02$ & $0.05 \pm 0.02$ & $0.04 \pm 0.02$ & $0.04 \pm 0.02$ & $1.78 \pm 0.07$ & $1.84 \pm 0.06$ \\
\hline & & High & $30.71 \pm 3.01$ & $32.29 \pm 2.45$ & $0.05 \pm 0.01$ & $0.05 \pm 0.01$ & $0.02 \pm 0.00$ & $0.03 \pm 0.01$ & $1.83 \pm 0.05$ & $1.82 \pm 0.06$ \\
\hline & NoLoad & Low & $33.76 \pm 3.50$ & $34.42 \pm 3.68$ & $0.07 \pm 0.04$ & $0.06 \pm 0.03$ & $0.05 \pm 0.03$ & $0.05 \pm 0.03$ & $1.78 \pm 0.07$ & $1.81 \pm 0.09$ \\
\hline & Overhead & Medium & $31.44 \pm 2.86$ & $32.35 \pm 4.06$ & $0.06 \pm 0.02$ & $0.06 \pm 0.03$ & $0.04 \pm 0.02$ & $0.04 \pm 0.02$ & $1.82 \pm 0.04$ & $1.81 \pm 0.07$ \\
\hline & & High & $30.59 \pm 2.98$ & $30.47 \pm 2.71$ & $0.04 \pm 0.01$ & $0.05 \pm 0.02$ & $0.02 \pm 0.01$ & $0.02 \pm 0.01$ & $1.83 \pm 0.06$ & $1.83 \pm 0.07$ \\
\hline \multirow[t]{5}{*}{ Uphill } & Back & Medium & $35.03 \pm 3.77$ & $38.89 \pm 2.95$ & $0.05 \pm 0.02$ & $0.11 \pm 0.08$ & $0.04 \pm 0.02$ & $0.08 \pm 0.04$ & $1.79 \pm 0.06$ & $1.74 \pm 0.06$ \\
\hline & & High & $33.97 \pm 2.02$ & $37.37 \pm 2.83$ & $0.06 \pm 0.02$ & $0.08 \pm 0.02$ & $0.04 \pm 0.01$ & $0.06 \pm 0.02$ & $1.79 \pm 0.06$ & $1.77 \pm 0.07$ \\
\hline & NoLoad & Low & $35.87 \pm 3.40$ & $38.47 \pm 3.27$ & $0.06 \pm 0.02$ & $0.10 \pm 0.04$ & $0.05 \pm 0.01$ & $0.08 \pm 0.04$ & $1.78 \pm 0.09$ & $1.75 \pm 0.07$ \\
\hline & Overhead & Medium & $32.85 \pm 3.37$ & $37.89 \pm 3.34$ & $0.05 \pm 0.01$ & $0.09 \pm 0.04$ & $0.03 \pm 0.01$ & $0.07 \pm 0.03$ & $1.83 \pm 0.05$ & $1.75 \pm 0.08$ \\
\hline & & High & $31.60 \pm 3.62$ & $35.37 \pm 2.82$ & $0.05 \pm 0.02$ & $0.06 \pm 0.02$ & $0.02 \pm 0.01$ & $0.04 \pm 0.01$ & $1.80 \pm 0.08$ & $1.78 \pm 0.06$ \\
\hline
\end{tabular}




\section{References}

Baloh, R. W., S. Spain, T. M. Socotch, K. M. Jacobson and T. Bell (1995). "Posturography and balance problems in older people." J Am Geriatr Soc 43(6): 638-644.

Bhambhani, Y. and R. Maikala (2000). "Gender differences during treadmill walking with graded loads: biomechanical and physiological comparisons." Eur J Appl Physiol 81(1-2): 75-83.

Birrell, S. A. and R. A. Haslam (2009). "The effect of military load carriage on 3-D lower limb kinematics and spatiotemporal parameters." Ergonomics 52(10): 1298-1304.

Bloom, D. and A. P. Woodhull-Mcneal (1987). "Postural adjustments while standing with two types of loaded backpack *." Ergonomics 30(10): 1425-1430.

BLS (2014-2015). Bureau of Labor Statistics.

Borg, F. G. and G. Laxaback (2010). "Entropy of balance - some recent results." Journal of NeuroEngineering and Rehabilitation 7(1): 38.

Chaffin, D. B. and G. B. J. Anderson (1984). Occupational Biomechanics, Wiley.

Cham, R. and M. S. Redfern (2002). "Heel contact dynamics during slip events on level and inclined surfaces." Safety Science 40(7-8): 559-576.

Chansirinukor, W., D. Wilson, K. Grimmer and B. Dansie (2001). "Effects of backpacks on students: Measurement of cervical and shoulder posture." Australian Journal of Physiotherapy 47(2): 110-116.

Chow, D. H. K., M. L. Y. Kwok, J. C. Y. Cheng, M. L. M. Lao, A. D. Holmes, A. Au-Yang, F. Y. D. Yao and M. S. Wong (2006). "The effect of backpack weight on the standing posture and balance of schoolgirls with adolescent idiopathic scoliosis and normal controls." Gait \& Posture 24(2): 173-181.

Chow, D. H. K., M. F. Li, A. Lai and M. H. Pope (2011). "Effect of load carriage on spinal compression." International Journal of Industrial Ergonomics 41(3): 219-223.

Cromwell, R. L. (2003). "Movement strategies for head stabilization during incline walking." Gait Posture 17(3): 246-253.

Damavandi, M., P. C. Dixon and D. J. Pearsall (2012). "Ground reaction force adaptations during crossslope walking and running." Human Movement Science 31(1): 182-189.

Donker, S. F., A. Ledebt, M. Roerdink, G. J. Savelsbergh and P. J. Beek (2008). "Children with cerebral palsy exhibit greater and more regular postural sway than typically developing children." Exp Brain Res 184(3): 363-370.

Donker, S. F., M. Roerdink, A. J. Greven and P. J. Beek (2007). "Regularity of center-of-pressure trajectories depends on the amount of attention invested in postural control." Exp Brain Res 181(1): 1-11.

Emerich, R. Effect of Roof Inclination on Postural Stability and perceived sense of fall. Abstract of the Annual American Industrial Hygiene Conference and Exposition. Fairfax, VA, AIHA. 104.

Fernie, G. R., C. I. Gryfe, P. J. Holliday and A. Llewellyn (1982). "The relationship of postural sway in standing to the incidence of falls in geriatric subjects." Age Ageing 11(1): 11-16.

Frames, C., R. Soangra and T. E. Lockhart (2013). "Assessment of postural stability using inertial measurement unit on inclined surfaces in healthy adults - biomed 2013." Biomed Sci Instrum 49: 234242.

Gauchard, G. C., P. Lascombes, M. Kuhnast and P. P. Perrin (2001). "Influence of Different Types of Progressive Idiopathic Scoliosis on Static and Dynamic Postural Control." Spine 26(9): 1052-1058.

Gillen, M., J. A. Faucett, J. J. Beaumont and E. McLoughlin (1997). "Injury severity associated with nonfatal construction falls." Am. J. Ind. Med. 32(6): 647-655.

Goh, J. H., A. Thambyah and K. Bose (1998). "Effects of varying backpack loads on peak forces in the lumbosacral spine during walking." Clinical Biomechanics 13(1): S26-S31.

Goodgold, S., K. Mohr, A. Samant, T. Burns, T. Parke and L. Gardner (2001). "TRUNK FORWARD LEAN DURING BACKPACK USAGE UNDER VARYING LOAD AND TASK DEMANDS: PILOT FINDINGS OF TWO BOYS." Pediatric Physical Therapy 13(4): 196.

Grimmer, K., B. Dansie, S. Milanese, U. Pirunsan and P. Trott (2002). BMC Musculoskeletal Disorders 3(1): 10. 
Heglund, N. C., P. A. Willems, M. Penta and G. A. Cavagna (1995). "Energy-saving gait mechanics with head-supported loads." Nature 375(6526): 52-54.

Holbein, M. A. and M. S. Redfern (1997). "Functional stability limits while holding loads in various positions." Int J Ind Ergon 19(5): 387-395.

Hong, Y. and C.-K. Cheung (2003). "Gait and posture responses to backpack load during level walking in children." Gait \& Posture 17(1): 28-33.

Hong, Y. and J. X. Li (2005). "Influence of load and carrying methods on gait phase and ground reactions in children's stair walking." Gait \& Posture 22(1): 63-68.

Kang, J., E. C. Chaloupka, M. A. Mastrangelo and J. R. Hoffman (2002). "Physiological and biomechanical analysis of treadmill walking up various gradients in men and women." European Journal of Applied Physiology 86(6): 503-508.

Kinoshita, H. (1985). "Effects of different loads and carrying systems on selected biomechanical parameters describing walking gait." Ergonomics 28(9): 1347-1362.

Kinoshita, H. (2007). "Effects of different loads and carrying systems on selected biomechanical parameters describing walking gait." Ergonomics 28(9): 1347-1362.

Kirby, R. L., N. A. Price and D. A. MacLeod (1987). "The influence of foot position on standing balance." Journal of Biomechanics 20(4): 423-427.

Knapik, J., E. Harman and K. Reynolds (1996). "Load carriage using packs: a review of physiological, biomechanical and medical aspects." Appl Ergon 27(3): 207-216.

Lajoie, Y., N. Teasdale, C. Bard and M. Fleury (1993). "Attentional demands for static and dynamic equilibrium." Exp Brain Res 97(1): 139-144.

Lajoie, Y., N. Teasdale, C. Bard and M. Fleury (1996). "Upright standing and gait: are there changes in attentional requirements related to normal aging?" Exp Aging Res 22(2): 185-198.

Ledin, T. and L. M. Odkvist (1993). "Effects of increased inertial load in dynamic and randomized perturbed posturography." Acta Otolaryngol 113(3): 249-252.

Leroux, A., J. Fung and H. Barbeau (2002). "Postural adaptation to walking on inclined surfaces: I. Normal strategies." Gait \& Posture 15(1): 64-74.

Lichtenstein, M. J., S. L. Shields, R. G. Shiavi and M. C. Burger (1988). "Clinical determinants of biomechanics platform measures of balance in aged women." J Am Geriatr Soc 36(11): 996-1002.

Lipsitz, L. A. and A. L. Goldberger (1992). "Loss of 'complexity' and aging. Potential applications of fractals and chaos theory to senescence." JAMA 267(13): 1806-1809.

Lloyd, R., B. Parr, S. Davies, T. Partridge and C. Cooke (2010). "A comparison of the physiological consequences of head-loading and back-loading for African and European women." European Journal of Applied Physiology 109(4): 607-616.

Maki, B. E., P. J. Holliday and G. R. Fernie (1990). "Aging and postural control. A comparison of spontaneous- and induced-sway balance tests." J Am Geriatr Soc 38(1): 1-9.

Martin, P. E. and R. C. Nelson (1986). "The effect of carried loads on the walking patterns of men and women." Ergonomics 29(10): 1191-1202.

Minetti, A. E., F. Formenti and L. P. Ardigo (2006). "Himalayan porter's specialization: metabolic power, economy, efficiency and skill." Proceedings of the Royal Society B: Biological Sciences 273(1602): 27912797.

Muslim, K. and M. A. Nussbaum (2015). "Traditional posterior load carriage: effects of load mass and size on torso kinematics, kinetics, muscle activity and movement stability." Ergonomics: 1-13.

Nag*, P. K. and R. N. Sen (1979). "Cardio-respiratory performance of porters carrying loads on a treadmill." Ergonomics 22(8): 897-907.

Noble, J. W. and S. D. Prentice (2008). "Intersegmental coordination while walking up inclined surfaces: age and ramp angle effects." Exp Brain Res 189(2): 249-255.

Pai, Y. C. and J. Patton (1997). "Center of mass velocity-position predictions for balance control." J Biomech 30(4): 347-354.

Pascoe, D. D., D. E. Pascoe, Y. T. Wang, D.-M. Shim and C. K. Kim (1997). "Influence of carrying book bags on gait cycle and posture of youths." Ergonomics 40(6): 631-640. 
Perry, J., S. k and J. R. Davids (1992). "Gait Analysis." Journal of Pediatric Orthopaedics 12(6): 815.

Prieto, T. E., J. B. Myklebust, R. G. Hoffmann, E. G. Lovett and B. M. Myklebust (1996). "Measures of postural steadiness: differences between healthy young and elderly adults." IEEE Trans Biomed Eng 43(9): 956-966.

Redfern, M. S., R. Cham, K. Gielo-Perczak, R. Grönqvist, M. Hirvonen, H. Lanshammar, M. Marpet, C. Y.-C. Pai and C. Powers (2001). "Biomechanics of slips." Ergonomics 44(13): 1138-1166.

Richman, J. S., D. E. Lake and J. R. Moorman (2004). "Sample Entropy." 384: 172-184.

Roerdink, M., P. Hlavackova and N. Vuillerme (2011). "Center-of-pressure regularity as a marker for attentional investment in postural control: a comparison between sitting and standing postures." Hum Mov Sci 30(2): 203-212.

S. Redfern, M. and J. DiPasquale (1997). "Biomechanics of descending ramps." Gait \& Posture 6(2): 119-125.

Simeonov, P. I., H. Hsiao, B. W. Dotson and D. E. Ammons (2003). "Control and Perception of Balance at Elevated and Sloped Surfaces." hfes 45(1): 136-147.

Simeonov, P. I., H. Hsiao, B. W. Dotson and D. E. Ammons (2003). "Control and perception of balance at elevated and sloped surfaces." Hum Factors 45(1): 136-147.

Smith, B., K. M. Ashton, D. Bohl, R. C. Clark, J. B. Metheny and S. Klassen (2006). "Influence of carrying a backpack on pelvic tilt, rotation, and obliquity in female college students." Gait \& Posture 23(3): 263267.

Spirduso, W. W. and L. A. Asplund (1995). "Physical-Activity and Cognitive Function in the Elderly." Quest 47(3): 395-410.

Stergiou, N. (2004). Innovative Analyses of Human Movement, Human Kinetics.

Stins, J. F., M. E. Michielsen, M. Roerdink and P. J. Beek (2009). "Sway regularity reflects attentional involvement in postural control: effects of expertise, vision and cognition." Gait Posture 30(1): 106109.

Sun, J. I. E., M. Walters, N. Svensson and D. Lloyd (1996). "The influence of surface slope on human gait characteristics: a study of urban pedestrians walking on an inclined surface." Ergonomics 39(4): 677-692.

Teasdale, N., C. Bard, J. LaRue and M. Fleury (1993). "On the cognitive penetrability of posture control." Exp Aging Res 19(1): 1-13.

Thomas, C. K., R. B. Stein, T. Gordon, R. G. Lee and M. G. Elleker (1987). "Patterns of reinnervation and motor unit recruitment in human hand muscles after complete ulnar and median nerve section and resuture." Journal of Neurology, Neurosurgery \& Psychiatry 50(3): 259-268.

Thurmon E Lockhart, J. L. (2013). "Aging Effect on Foot Dynamics during Unexpected Slips." Clinical Research on Foot \& Ankle 01(02).

Vuillerme, N., N. Forestier and V. Nougier (2002). "Attentional demands and postural sway: the effect of the calf muscles fatigue." Med Sci Sports Exerc 34(12): 1907-1912.

Vuillerme, N., B. Isableu and V. Nougier (2006). "Attentional demands associated with the use of a light fingertip touch for postural control during quiet standing." Exp Brain Res 169(2): 232-236.

Vuillerme, N. and V. Nougier (2004). "Attentional demand for regulating postural sway: the effect of expertise in gymnastics." Brain Res Bull 63(2): 161-165.

Wade, L., W. Weimar and J. Davis (2004). "Effect of personal protective eyewear on postural stability." Ergonomics 47(15): 1614-1623.

Yeoh, H. T., T. E. Lockhart and X. Wu (2013). "Nonfatal Occupational Falls Among U.S. Health Care Workers, 2008-2010." Workplace Health Saf 61(1): 3-8. 